\title{
La problemática de género en contexto de marginalidad. Lecturas de una experiencia. ${ }^{1}$
}

\section{The gender problematics in context of marginality. Readings of an experience.}

\author{
Analía Tannuri²
}

\section{Resumen}

Este artículo analiza la experiencia producida por la implementación de un dispositivo grupal para mujeres dentro de un centro cultural barrial de la periferia de la ciudad de Rosario. Se toma como núcleo el dispositivo construido, las lecturas institucionales y políticas previas y los efectos posteriores a nivel subjetivo, grupal e institucional, con especial hincapié en los movimientos subjetivantes producidos en el transcurso del pasaje de las mujeres por el espacio. Asimismo, se pone en evidencia la demanda de inclusión política e institucional de determinados aspectos de la subjetividad femenina en contextos de marginalidad que quedan por fuera de circuito, ya sea por expulsión o invisibidad.

Palabras Clave: mujeres - dispositivo grupal - efectos subjetivantes - inclusión - género

\section{Abstract}

This article analyzes the experience produced by the creation of a grupal system for women in a cultural center located in the periphery of Rosario's city. The analyzers of the system are: the institutional and previous policies analysis, the subjective, group and institutional later effects; we specially take into consideration the subjectifying movements occurred during the work of the women in the system. We likewise underline the demand of political and institutional incorporation of certain aspects of the feminine subjectivity in contexts of marginality that are not considered and therefore, stay out of the system due to either expulsion or invisibility.

Keywords: women - group system subjective effects - inclusion - gender

\section{Introducción}

En este trabajo nos proponemos abordar la experiencia de un taller de mujeres realizada con un equipo de profesionales de diferentes campos (Trabajo Social, Medicina, Psicología) que nos encontrábamos, como residentes algunas y como militantes otras, en un centro cultural barrial ubicado en la periferia de la ciudad de Rosario entre los años 2011 y 2012.

\footnotetext{
${ }^{1}$ Artículo escrito sobre la base del trabajo final integrador para la Carrera de Especialización en Psicología Clínica, Institucional y Comunitaria de la Universidad Nacional de Rosario. El TIF, dirigido por Ps. Claudio Cúneo, fue presentado en 2014 y aprobado en 2015.

${ }^{2}$ Psicóloga. Especialista en Psicología Clínica, Institucional y Comunitaria (UNR). Docente de la Cátedra Introducción a la perspectiva de género de la Carrera de Psicología (UNR). Activista Feminista dentro de la colectiva Mala Junta, a cargo del equipo de violencia y la coordinación de espacios grupales de mujeres en situación de violencia desde el año 2015.
} 
Hasta ese momento, la demanda de la institución se relacionaba con el imperativo de llevar a cabo acciones que resolvieran lo que desde la coordinación se formulaba como problema. Por ejemplo, lograr que los niños y niñas de determinado asentamiento concurrieran a la escuela de manera regular. Pero había una queja que no llegaba a tomar forma de demanda, más bien se mostraba como una molestia con respecto a que un grupo de mujeres no conseguía incluirse en las propuestas que se ofrecían, en general con la forma de talleres semestrales o anuales provenientes de programas del gobierno de la provincia. Estas mujeres eran madres de niños y niñas que concurrían a algún espacio dentro del centro cultural. La queja tenía que ver con cierta incomodidad acerca del estar de estas mujeres sin hacer nada, es decir, sin incluirse en la modalidad de asistente a un taller, o reconocerse como alumna de determinado profesor.

La experiencia que se toma para analizar surge de una práctica con un objetivo político: la visibilización de la problemática de género. Dichas inquietudes emergieron del anudamiento entre la condición de residente por la carrera en un Centro de salud del barrio y la militancia feminista dentro del centro cultural del mismo barrio. Pero la continuidad del espacio, la intimidad, la circulación de la palabra, el tiempo para la elaboración del sufrimiento y el surgimiento de nuevos modos de narrar y distintos modos de creación se revelaron con posterioridad como efectos que excedieron este objetivo primero, como así también los posteriores impactos en las configuraciones institucionales y colectivas.

A partir de esta experiencia se generaron una serie de convergencias e interrogantes acerca de la problemática de género que afectaba a mujeres de sectores marginalizados y la forma que tomaba su padecimiento. Nos interrogábamos - como equipo de trabajo- acerca de los modos en que ellas actuaban dentro de la institución aquello que no tenía palabras para ser dicho, el lugar postergado que ocupaban. Se trataba de aquello que aparecía como molestia, pero más tarde entendimos que se trataba de una resistencia indomesticable a las formas de tratamiento que se ofrecían. Resistencia eficaz porque permitió que ese circular en desventaja no se naturalizara, es decir, que no pasara sin ser visto.

Este escrito se propone reflexionar sobre el espacio grupal como dispositivo que posibilita el tratamiento de este malestar. Se intentarán situar, además, los efectos en el nivel de la singularidad, de lo institucional y de lo colectivo.

\section{Dimensiones política e institucional del problema}

Cuando se propuso el armado del taller, la institución lo aceptó de inmediato con el pedido que se hiciera algo con ellas. Todo parecía muy factible ya que la no-inclusión de esta población generaba incomodidad a la institución y la propuesta del espacio fue recibida como una solución. Pero había una diferencia inicial en el modo de formulación de la problemática. El taller no planeaba un hacer prefigurado para ellas, sino que propiciaba un espacio para que ellas bicieran algo con eso que se ofrecía. Se las habilitaba como sujetas de acción -no como objetos sobre los cuales accionar. Esta diferencia fue escuchada luego de iniciado el espacio, sobre todo a partir de la incomodidad que generaba la irrupción de la coordinadora de la institución en el taller. Desde la coordinación del taller ubicábamos el problema en estas propuestas genéricas que masifican, en tanto no alojan la singularidad. Esta demanda de hacer algo con ellas, así como se hacía algo con la población de determinado asentamiento, seguía ubicándolas como obje- 
to de intervención. Ese con, paradójicamente, las excluía. Y ese modo de hacer con aquel malestar que circulaba por fuera de las propuestas genéricas, para el equipo de trabajo que constituíamos se reveló como la condición de reproducción del hacer institucional, la dimensión de lo instituido.

La institución dejó de ver a estas mujeres, naturalizó su circulación y esto produce como síntoma la aparición de lecturas que las ubican en un lugar de objeto a tutelar o reeducar dando una respuesta automática frente a la dificultad de recibirlas y escucharlas en su diferencia.

Ubicamos aquí un síntoma de la institución, entendiendo que al no tener un espacio al cual llegar, aparece en el lugar del problema algo que molesta y con lo que no se puede hacer nada porque la institución no se ve concernida. Es cierto que ellas no podían incluirse en las propuestas institucionales, que las bordeaban, las rondaban, cebaban mate alrededor de los talleres, no bailaban reggaeton ni iban a carpintería, pero estaban allí en el medio, pasando la escoba o el trapo, ni adentro ni afuera, molestando. La lectura de la institución era que estaban en el borde, como cayéndose.

Un tratamiento posible del problema así formulado surgía a través de la lectura deficitaria. Muy comúnmente desde el discurso pediátrico se indicaba un acompañamiento terapéutico para alguna mujer porque "no llegaba a ser la madre que se esperaba que fuera". Entonces podían tener un acompañante que las ayudara a ser madres. Es decir, un actor o actora social enunciaba como problema la maternidad deficitaria y el Estado disponía recursos para que esta mujer alcanzara al menos un estándar. ${ }^{3}$

\footnotetext{
${ }^{3}$ Se hicieron numerosos pedidos de acompañamiento en esos años para lograr inscribir dentro de las tareas maternas, la obligación de que los hijos e hijas sostuvieran la escuela. Muchas mujeres no lograban comprender la importancia de la escolaridad
}

Pero no se podía tener un acompañante por los efectos del aislamiento y la vulnerabilización que sufrían por el hecho de ser mujeres, por la falta de autonomía a la que las había arrojado el proyecto familiar y las violencias cotidianas a las que estaban sometidas, relegadas a la crianza de sus hijos e hijas, aisladas de todo tipo de red de sociabilidad y cada vez más dependientes de la dinámica familiar, sin un proyecto propio por fuera de ese. La lectura que hicimos desde el equipo de trabajo es que esta dimensión no constituía un problema para los actores y actoras sociales en aquel campo. Su condición de mujeres permanecía por fuera de la visión estatal. Estas personas sólo eran vistas en tanto "madres" o "pobres", lugar desde el cual sólo se esperaba de ellas la disposición pasiva a recibir recursos, con sus consecuentes efectos desubjetivantes. Paradójicamente, su autoevidente condición genérica resultaba una categoría invisible para los marcos epistémicos de la institución (Thiers Vidal, 2002).

Dice al respecto Ana María Fernández (2014):

Los procesos de inferiorización, discriminación y fragilización operan como naturalizaciones;

conforman en tal sentido invisibles sociales. (...) Un invisible social no es algo oculto o escondido, sino que -paradójicamente- se conforma de hechos, acontecimientos, procesos y dispositivos reproducidos en toda la extensión de la superficie social y subjetiva. Está ahí, pero no se ve o se lo considera natural. (p 33)

porque ellas mismas no habían pasado por eso, o bien lo habían hecho de manera muy irregular y como consecuencia tenían, ellas mismas, una débil inscripción institucional. 
Nuestra lectura es que, aunque la institución las expulsaba, allí resistían, en los bordes. Pensamos que este modo de resistir tiene que ver con las relaciones del sujeto con el poder, tema del que se ocupa Gilou García Reinoso (2000), más precisamente las relaciones del sujeto con el gran Otro en sus formas históricas. La autora entiende que el poder, en el lugar de Amo, emite discurso y propone identificaciones, ligando el deseo a las representaciones que se le ofrecen, con lo cual consigue el consenso, apoyándose en los procesos más arcaicos de la constitución subjetiva. Ella propone que las relaciones que mantenga el sujeto con el discurso político tendrán las marcas de las relaciones inconscientes del sujeto con el Otro. En este modo de circular en los bordes leemos un modo de resistencia, loco y sintomático por la incomodidad que produce, pero también efectivo, de no ser capturadas por las lógicas patriarcales que producen el recorte del problema, y las lógicas manicomializantes que las ubican en lugar de objeto. Esta lectura relanza la posición analítica para desmontar las relaciones del sujeto al poder y hacer algo nuevo con eso. De aquí surge la idea de armar grupo para tramitar algo del malestar de manera colectiva, para interrogar el síntoma y hacerlo hablar.

Entonces comenzamos a recortar un problema, a pensar en sus diferentes dimensiones $y$ a ofrecer una instancia donde fuera posible formular de qué se trataba eso que insistía y molestaba.

Muchas de ellas vivían en condiciones muy precarias, en una mala vivienda que aloja a varias generaciones, en malas condiciones sanitarias, escasamente escolarizadas, con una débil referencia a las instituciones.

Para estas mujeres con las que trabajamos, el aislamiento era un destino al que las expulsaba su condición de pobres, pero además de mujeres, ya que esto implicaba que, dentro del circuito de la pobreza y marginalidad, además estaban conminadas a las tareas de reproducción de la vida hogareña y de reproducción biológica. En la institución repetían la misma escena que en la vida privada: mantenerse al margen de todo tipo de intercambio productivo. Destino apropiado y convertido en síntoma que molesta, que pica, y que al ser interrogado muestra eso que no tiene palabras para ser dicho dentro de las limitaciones epistémicas estructurales (Thiers Vidal, 2002). Esto permite, de ahí en más, comenzar un trabajo de nominación y luego un pasaje de la anécdota a la categoría (Amorós, 2005).

Como plantea Ana María Fernández (2014), para que un grupo sea discriminado es necesario que haya un proceso de apropiación, tanto de los bienes producidos por éste como de las potencias que como grupo tiene para producir esos bienes. Bienes como la capacidad para dar cuidados, para reconfortar la fuerza de trabajo, para limpiar y mantener la vivienda como un espacio habitable, cuidar de la niñez, etc. Esta doble apropiación garantiza que circulen por los mismos circuitos, pero con desigualdad de oportunidades y que esta circulación en desventaja se despliegue sin ser vista.

\section{Diálogos entre el Psicoanálisis y la problemática de género}

Es oportuno retomar el planteo de Débora Tajer (2013) en relación al sentido que ha producido la introducción de la perspectiva de género en el trabajo y pensamiento psicoanalítico. La autora señala que justamente el punto de partida de los estudios de género en diálogo con el Psicoanálisis se encuentra en el intento de relacionar el estado subordinado de las mujeres en el sistema patriarcal con las formas del malestar femenino. La in- 
dagación se realizó siguiendo la línea de Freud (1930) en "El malestar en la cultura", texto en el que expresa que no se puede esperar una conformación similar en los aspectos morales de aquellas personas que más gozan de los bienes culturales y sociales, y aquellos sectores más pobres. Freud se refería a los sectores más acomodados económicamente. Hoy, a partir de los desarrollos de reconocidas intelectuales respecto del Patriarcado (Millet, 1975; Rubin, 1975; Firestone, 1970; Guillaumin, 1972), se puede pensar en los privilegios que otorga la pertenencia a determinado género. Desde los Estudios de Género se retoman estos desarrollos como herramientas para visibilizar un grupo que no había sido pensado como subordinado socialmente: las mujeres, hasta entonces consideradas como constituyendo su psiquismo en tanto efecto psíquico de la diferencia sexual anatómica. Esta creciente receptividad de los distintos campos de las ciencias sociales y humanas respecto de la teoría feminista y los estudios de género permitió correr la feminidad del campo de la esencia y de la determinación psíquica de la biología para darle un estatus de construcción social que constituye psiquismo.

Tratándose de un concepto polisémico cuyo sentido es objeto de discusiones en el campo de la teoría social y los estudios de género, optaré por una reposición del término Patriarcado dentro del pensamiento de Kate Millet (1975), una de las mayores exponentes del feminismo radical estadounidense, quien en su tesis "Política sexual" inaugura la historia de este concepto dentro de la teoría y praxis feminista. Ella dice:

El ejército, la industria, la tecnología, las universidades, la ciencia, la política y las finanzas - en una palabra, todas las vías del poder, incluida la fuerza coercitiva de la policía - se hallan enteramente en manos masculinas. $\mathrm{Y}$ como la esencia de la política radica en el poder, es infalible el impacto de semejante privilegio (...) así como también los valores, la ética, la filosofía y el arte de nuestra cultura son también de fabricación masculina (...) Si consideramos al gobierno patriarcal como una institución en virtud de la cual una mitad de la población (es decir, las mujeres) se encuentra bajo el control de la otra mitad (los hombres), descubrimos que el Patriarcado se apoya sobre dos principios fundamentales: el macho ha de dominar a la hembra, y el macho de más edad ha de dominar al más joven (p 70).

Otro aporte indispensable de los Estudios de Género al Psicoanálisis se encuentra en la articulación y problematización de las posibles retroalimentaciones entre una analítica del poder y del sujeto, o entre las relaciones de dominación y los modos de subjetivación en sus respectivos cruces con el clivaje sexo-genérico, complejizando el planteo de Freud sobre el poder como un problema para la psicología circunscripto originalmente a la asimetría entre generaciones. En este sentido, los Estudios de Género ayudaron a identificar relaciones de dominación que exceden las jerarquías intergeneracionales, ofreciendo herramientas para un abordaje más integral de las problemáticas vinculadas a este tipo de padecimientos.

En esta línea de planteos atinentes a las relaciones del sujeto al poder se podría decir que la estrategia de vulnerabilización (Fernández, 2013) específica que tiene por receptor a este grupo poblacional en contextos de marginalización, apunta al aislamiento, la apatía, el quiebre de los soportes relacionales y la invisibilización de la problemática. 
Las vecinas que concurrían al centro cultural estaban ahí, circulaban, cebaban mate, limpiaban. Estaban ahí pero no tenían un lugar. Ya se conocían sus historias (clínicas) familiares, ya no había asombro, se habían vuelto invisibles a menos que quisieran consultar por su Salud Mental. En la institución las llamaban "mujeres vulnerables". Nadie se preguntaba por qué o cómo llegaron hasta ahí, qué pasó en la historia de estas mujeres. Ellas tampoco hablaban. El discurso de género sirvió para interrogar esto. El Psicoanálisis permitió pensar que había que poner en movimiento algo de esta situación cristalizada a partir de hacer circular la palabra, generar inscripciones. No había ningún tratamiento de esta población más que la queja, no había lugar desde el cual esperarlas, ni modo de alojarlas. Las categorías disponibles para pensar la problemática sostenían prácticas tutelares que eran resistidas por ellas porque provenían de un lugar de control, apuntaban a normalizarlas, para que llegasen a alcanzar algún estándar. Esto hacía muy difícil la relación con ellas, ya que tendían a desconfiar y a rechazar cualquier intervención que viniera desde el Estado. Nos preguntaban: "Ustedes de dónde vienen? ¿Quién las manda? ¿Cuánto tiempo se van a quedar?"

La hipótesis de trabajo del equipo apuntó a lo grupal, pensando que podía funcionar como dispositivo para el empoderamiento, ya que justamente apunta a armar red de sostén, que va a contramano del aislamiento que producen los dispositivos de vulnerabilización. Porque al encontrarse, hablar con otras de aquello que les pasa, ven que no son las únicas, que a las demás les pasa algo parecido y pueden escucharlo en un espacio contenedor, donde la imagen que les vuelve de la otra no es la del desconocimiento, sino una que puede contrarrestar u ofrecer alternativas de resistencia a las mencionadas estrategias de vulnerabilización.

Consideramos oportuno recuperar de la praxis feminista la herramienta de los grupos de autoconciencia, espacios donde las mujeres se encuentran hace más de medio siglo dejando como legado la ejercitación de la toma de la palabra, la validación y politización de la experiencia propia gracias a la legitimación que propicia la escucha sensible y recíproca. Estas prácticas remiten directamente al objetivo que perseguían los grupos de autoconciencia durante la llamada segunda ola feminista, de hacer político lo personal. Con esta herramienta se logró relativizar el clivaje entre lo pólítico-público y lo privado, lo cual funciona como pivote para cuestionar los supuestos pretendidamente universales de los discursos emancipatorios para propiciar la politización de temas como el amor, la familia, la maternidad, los cuidados, la domesticidad, la heteronorma, la conyugalidad, la prostitución, la naturaleza, la biología, la cultura, los procesos de racialización y otros (Caudana, 2018).

\section{La propuesta: el dispositivo grupal}

Entre la multiplicidad de demandas que presentaba la institución, se define comenzar por resolver qué hacer con la cantidad de ropa que llegaba a través de donaciones, bajo la forma de Roperito. Entendíamos que dicho espacio era estratégico como puerta de entrada y como excusa para el encuentro, nos permitía estar allí sin caer en la tallerización y soportando el devenir.

La propuesta era organizar esa ropa y ponerla a la venta, mientras trabajábamos algunas cuestiones que tenían que ver con ellas y así comenzar a funcionar. Los encuentros eran de dos horas una vez por semana y coincidían con el Roperito. Cinco coordinadoras y unas seis vecinas rotábamos para atender. 
Una de las dificultades era sostener el adentro del espacio, no dejarse aplastar por la urgencia de la institución que entraba y salía todo el tiempo, amenazando la intimidad que el dispositivo precisaba para poder funcionar:

Algunas veces se acercaba la coordinadora del centro cultural al espacio y se sentaba entre nosotras, sin avisar que vendría y sin someterse a las reglas del espacio como son los horarios de inicio y finalización, el respeto de las tareas o la escucha de las compañeras. Eran momentos de tensión para todas, porque esta persona venía a pedir cuentas sobre la productividad del espacio, a recordarle a alguna que le había conseguido un subsidio; recordándoles a todas que iban allí a pedir: ayuda y subsidios. Esto iba a contramano de la lógica del espacio, puesto que no es lo mismo quedar en el lugar de alguien que pide, que convertirse en alguien que reconoce la presencia de recursos de los cuales puede disponer con distintos fines.

Esto resultaba muy disruptivo, pero no siempre provenía de afuera. Algunas veces, una de ellas se paraba para leer una producción escrita donde se animaba a hablar de sus sueños de bailar o ser escritora, de diseñar ropa, sus pensamientos reivindicativos de género, además de compartir con otras que había aprendido a leer y escribir y disfrutaba mucho de eso. Era un momento de mucho júbilo. Frecuentemente, frente a estos emergentes surgía un comentario dirigido a quien leía: "Che, ese que llora ¿no será tu hijo?”, recordándole a esa mujer en un momento de despliegue deseante las obligaciones maternas, la maternidad como obligación, como destino. Desde la coordinación se animaban las producciones y se procedió a convocar al profesor de Literatura y cuentos para que coordinara un espacio paralelo con los niños y niñas. Con el transcurso del tiempo, la atención del Roperito fue quedando al margen, deconstruyendo paulatinamente el imperativo que compelía a priorizar cualquier demanda que viniera de afuera, independientemente de su tiempo y oportunidad.

Los primeros momentos del taller no fueron tan jubilosos. Las historias de estas mujeres eran desgarradoras. Padres incestuosos, maltratos y violencias desde niñas, hombres que ejercían violencias y abusos físicos, ausencia de dispositivo de infancia como espacio para el juego tal y como era conocido por las coordinadoras que pertenecemos al grupo de mujeres blancas y burguesas. Lo que se intentaba desde el grupo era inscribir como políticas esas violencias cotidianas a las que habían sido sometidas desde muy pequeñas. Es decir, producir un acto que las filiara al género, hacerles lugar para que existan en una historia que se escribe con mayúscula y excede la historia singular pero la contiene: la historia contada por mujeres desde el movimiento de mujeres. Historia que tiene un saber y modos de enunciar aquello por lo que ellas pasaron, decirles que allí existen y no hace falta que inventen palabras para nombrar lo innombrable. Quizás no sabíamos exactamente qué les había pasado, pero entendíamos que el hecho de que las mujeres sean la abrumadora mayoría de quienes sufren violencia por parte de hombres significa que hay un poder social y subjetivo que es patrimonio del género masculino en este sistema patriarcal, y que muchos deciden ejercerlo bajo las formas públicas y privadas del abuso (Fernández, 2014).

Frente a estas marcas de desmantelamiento subjetivo es difícil pensar los modos institucionales de incluir. Nos encontramos con personas que no hablaban, que no pedían, que parecían encontrarse en una posición cristalizada de sufrimiento. Frente a esta realidad pensamos en un dispositivo como el grupal, con la capacidad de metabolizar (Montoya, Crisalle y Grande, 2011), es decir de poner en 
palabras, generando inscripciones posibilitadoras del armado de un relato. La posibilidad de nombrar algo del sufrimiento se encontraba petrificada bajo las formas de la naturalización.

Luego de un año de taller, estas mujeres que habían participado comenzaron a hablar, se preguntaban qué les pasaba a otras mujeres, participaron de movilizaciones colectivas que a su vez generaban más preguntas como ¿qué pensarán los hombres de esto? Comenzaron a escribir, se dirigieron a sus compañeras, luego a las talleristas y luego a todo el barrio desde un escenario con un micrófono - desde su lugar y en nombre propio- pero sintiéndose parte del Centro Cultural y con el respaldo del discurso feminista.

Por un lado, se trata del pasaje de lo privado y personal de las vivencias a algo de orden colectivo donde a otras les suceden cosas similares. Pero también se trata del tránsito por lo público donde sus derechos se inscriben en una trama colectiva. Lo anterior produjo un efecto filiatorio en el que nos interesa profundizar.

\section{Lo grupal, sus legalidades y sus efectos}

La invitación a formar parte de un grupo no implica pertenecer a una muchedumbre sino a un número discreto de personas. Además, hay una serie de reglas que requieren un acotamiento del goce para poder pertenecer. Es decir, hay que prepararse para llegar porque hay un horario acordado de comienzo y finalización, un sitio de trabajo seleccionado dentro de la institución, una se viste apropiadamente, se expone a la mirada de otros, hay alguien que convoca y se pone en escena cierta hospitalidad, hay una tarea, reglas para realizarla, todo lo cual arma una escena (Jasiner, 2011). El encuentro no transcurre de cualquier modo, hay un encuadre metodológico que establece ciertas constantes que se repiten y ordenan la escena, pero a la vez hay espacio para la creación, dentro de determinado marco. Hay que mantener un orden para hablar, conversar de un tema y no de otro, respetar el tema que se propone por encuentro, son cuestiones que hacen a un orden simbólico. Dentro de la continuidad del tiempo y el espacio institucional se instala una marca diferenciadora. Hay un equipo de coordinadoras que instalan un ritmo de encuentros, un espacio-tiempo propio creado para el dispositivo: el horario, el día y el espacio que se utiliza. Se genera entonces un tiempo de ilusión compartida y de satisfacción cuando se crea con otros, aparecen nuevas respuestas y lugares posibles para habitar.

Luego de un tiempo de trabajo dejaron de insistir los relatos del horror para dar lugar al trabajo sobre algún contenido. Pareciera que aquellos afectos del miedo, la culpa y la vergüenza encontraron nuevas formas de expresión. Se propició la escritura de textos de reivindicación en los que se reflejaba un ejercicio del pensamiento para transmitir preocupaciones colectivas que habían encontrado en el taller un espacio y un tiempo para enunciarse y dirigirse a un interlocutor. Entonces aquel exceso de realidad se volvió creación. Aparecieron los dibujos, la invención de un logo propio con colores y significados asignados por ellas, que luego fue plasmado en la plaza del barrio por el profesor de Grafitti durante una intervención que servía como cierre del primer año de trabajo. También surgió la escritura de pequeñas ficciones para transmitir algo de sus historias singulares, que luego fueron plasmadas en siluetas anónimas y ofrecidas al público. Las otras instituciones del barrio y diferentes actores y actoras comunitarios se acercaron para acompañar y apoyar. Ese día un spot radial reproducía las voces en 
off de las mujeres, grabaciones realizadas para colectivizar fragmentos del taller, momentos de juego, de lectura y reflexión, intercalados con música que habían seleccionado para ese día. Algunas se animaron a subir al escenario, relatar por qué estábamos allí, presentar el espacio e invitar a otras mujeres del barrio. Muchos de sus compañeros y familiares no concurrieron al evento final donde se realizó la puesta en común del trabajo de todo el año. Pero aquello que habían escrito para compartir y que armamos para mostrar, las había transformado. Trabajo de sublimación pensado como otro destino posible para las pulsiones en el marco del trabajo grupal.

Acompañamos la transición entre quedar tomada por una experiencia dolorosa y luego tomar esta experiencia con otras para pensarla y transformarla colectivamente. Este modo de organizarse tuvo un impacto en la singularidad, pero, también, múltiples resonancias en las otras integrantes del grupo, en tanto pudieron apropiarse de otra faceta de la legalidad, que puede marcar límites a alguien que los desconoce, legitimar que allí se produjo un daño, que este acto tiene consecuencias y se puede exigir que sea reparado. Esto generó una nueva inscripción, una marca que se recuperó como colectiva y tuvo efectos reparadores en la singularidad, en tanto permitió instalar otras huellas más allá de la cultura de la mortificación (Ulloa, 2011). En esa situación tan adversa es posible juntarse, darse palabras. Que algunas pongamos en juego nuestro deseo de que todo esto surja posibilita que aparezcan otras en posición deseante.

Es decir, el grupo funcionó como espacio privilegiado de elaboración. Se propició un tiempo para pensar, frente al imperativo exterior de la acción. En dicha escena las coordinadoras limitamos las intervenciones a facilitar la circulación de la palabra o relanzar alguna frase en la que se hubiera tomado posición.

\section{E1 trazo singular dentro de la trama grupal}

Es oportuno relatar una situación particular que ocurrió durante el tiempo de trabajo en el espacio grupal para dar cuenta de los efectos en la subjetividad de la disposición deseante de las mujeres que integrábamos la coordinación, y cómo nuestra disposición amorosa contrarrestaba lo mortífero de las condiciones políticas y económicas que habían arrojado a algunas de ellas al desmantelamiento subjetivo.

Había una mujer entre las participantes del taller que durante los primeros encuentros venía muy desarreglada, a veces con la ropa sucia, no tenía dentadura y por eso sentía mucha vergüenza de hablar. Solamente cebaba mates y escuchaba, repitiendo en la escena grupal su posición en la institución. Muchas intervenciones que hacíamos desde la coordinación tenían que ver con el cuidado hacia ella, como ofrecer un espacio de juego para sus niños mientras ella trabajaba, estar atentas a sus posibilidades de llegar al espacio, además de aquellas otras cosas que hubiéramos querido hacer y no pudimos. Esas otras cosas que como mujeres deseábamos para ella se convirtieron en ese plus de las intervenciones que, como lugar de otro que sostiene, que arma, generó efectos incluso en el cuerpo de esta mujer. Luego de un tiempo pudo llegar vestida con ropa que ella misma seleccionaba, que tomaba del roperito o que "cirujeaba" (así lo decía). Ella le hacía arreglos, apliques, marcas que la diferenciaban como suya. También comenzó a fabricar bijouterie y a lucirla. Decidió tomar un turno para hacerse una dentadura (hacía tiempo se planteaba como un imperativo y una necesidad dentro del centro cultural al cual nunca podía llegar porque perdía los turnos). Ahora podía sonreír reinventando su imagen, mirándose en nuevos espejos que 
devolvían las miradas que acompañaban este cambio, invistiendo lo nuevo como parte del propio cuerpo. Ella pasó de un estado de silencio y perplejidad a otro en que pudo hablar para decirnos "yo no quería vivir más, pero con ustedes...”.

Gilou García Reynoso (1992) plantea que la organización de desigualdades tiene relación directa con el deseo de muerte del Otro encarnado en los discursos de poder. Estas condiciones objetivas de sufrimiento por acción de procesos políticos, económicos y sociales que condujeron a la desigualdad encuentran eco en el inconsciente con aquellas escenas de terror fundantes, a las que se anudan las vivencias de terror de las que fueron objeto estas mujeres en sus historias comunes. Esta es la inscripción psíquica según la autora, a través de la cual se legitima el sistema de dominación. En este punto plantea que muchas veces somos cómplices de la censura y no podemos pensar aquello inexplorado, aquello que aún carece de nombre, allí donde se levantan nuestras resistencias, porque lo que estamos cuestionando nos aplasta desde la exigencia de seguir sirviendo a los mismos amos y a título individual. Entonces, en ese espacio donde nos encontramos con otras para pensar estamos yendo en contra de estas exigencias, que cada vez se vuelven más feroces y nos generan culpa.

En el trabajo de pensar aquello inexplorado en el pequeño grupo, mediante la resonancia simbólica, se produce un deslizamiento entre lo que arma trama y el trazo singular a partir de que algo se inscriba. Un primer momento en el que un sujeto se proyecta imaginariamente sobre el otro que deviene pantalla, momento en que todas parecen iguales porque han vivido situaciones similares y un segundo momento donde se produce un registro a nivel de la singularidad, donde el mismo acontecimiento nunca se inscribe de la misma manera para dos sujetos. Retomando la idea de Piera Aulagnier (2010) podríamos pensar que el acceso a la narración, trabajo a través del significante, es lo que permitirá al sujeto una distancia progresiva respecto del goce traumático, pero cada una encontrará su manera.

La escena grupal propicia el instante de ver y el tiempo para pensar que no es sin otros (Jasiner, 2011) En el momento de concluir aparece la palabra, la reflexión que permite un corrimiento de la posición sacrificial convertida en destino para alguien en soledad. Es entonces cuando algunas cosas comienzan a ser enunciadas y dejan de ser invisibles: "todas hemos sufrido situaciones similares en nuestra vida, pero... ¿Cómo fue que cada una de nosotras se las ha arreglado para sobrevivir? ¿Cómo transcurrió esta historia? ¿Cómo es que llegamos a armar lo que hoy tenemos? ¿Cómo nos hemos arreglado con la maternidad? ¿Qué lugar tenemos en el centro cultural? ¿Qué somos para la coordinadora de la institución? ¿Por qué trabajamos sin cobrar? Preguntas muy valiosas para historizarse, pensarse, dejar de ser puro acto y elaborar algo a través de la palabra, en posición de sujeto y no de objeto de la historia. Todo esto tiene un efecto aliviador.

\section{Para concluir}

Al ser resistidas y desafiadas las normas por los mismos sujetos que son producidos por ellas, este trabajo de pensamiento y acción remueve muchas veces el sentimiento de culpa y, otras tantas, toma los caminos de la inhibición o el síntoma, obstaculizando la protesta, muchas veces conduciendo estas problemáticas al circuito de salud.

En el espacio institucional donde tuvo lugar la práctica analizada no se encontraban 
disponibles las herramientas para la escucha o para nombrar estos sucesos padecidos, o bien algún espacio para su tramitación, con lo cual quedaban conminados a la petrificación en el síntoma o algo muy parecido a lo que se realiza con la segregación institucional través de los discursos manicomializantes.

A su vez, las dificultades de esta población para acceder al circuito institucional tenían que ver con múltiples y diversos síntomas e inhibiciones que las habían alejado de los circuitos de lo público, quedando recluidas a lo doméstico; además de una serie de aprendizajes que tienen que ver con mandatos sociales destinados a garantizar la feminización de las tareas domésticas, reproductivas y de cuidado, lo que impide o demora, a veces durante muchos años, el acceso a la pregunta por el deseo propio. Si pusiéramos la mirada sobre ellas como responsables de no estar referenciadas a ninguna actividad, estaríamos desconociendo las vulneraciones de las cuales son objeto estos sectores de la sociedad, más aún si se trata de mujeres-madres, ocultando la desigualdad, enunciándola como discapacidad en nombre de una supuesta fragilidad mental.

¿Qué contrato proponen estas instituciones para las mujeres? ¿Qué lugares les ofrecen para ocupar? Esta es una de las primeras cuestiones con las que nos encontramos antes de plantear el espacio grupal. O acaso, luego de atravesar la experiencia, podríamos plantear que en el lugar del contrato o de un posible alojamiento algunas veces aparece la violencia institucional, allí donde hay ruptura del lazo social. Entonces la institución las señalaba como "en el borde" ubicando que no podían, que no llegaban, patologizando o culpabilizando. Esta posición institucional, sintomática de las rupturas del lazo social, también es un desafío para los dispositivos grupales que se crean dentro de tales instituciones, porque intentan resistir sin renegar el conflicto, trabajar en la tensión que implica pelear por la manera en que se conciben los proyectos, los modos en que se enuncian los problemas que muchas veces invisibilizan desigualdades y violencias. Ese trabajo se realiza apostando a la construcción de manera colectiva, subrayando que habrá puntos en los que estas mujeres estaban mortificadas, pero también hay otros en los que habían podido hacer algo con ese sufrimiento que traían. Las intervenciones apuntaban a fortalecerlas, acompañarlas en la búsqueda de sentido, a tomar algo de lo singular para construir lo colectivo.

Si pensamos, por ejemplo, en las conquistas del movimiento de mujeres en términos políticos y jurídicos, -el hecho de que las mujeres hayan conquistado derechos que las alejaran del tutelaje y las acercaran a la ciudadanía plena- entendemos que esto constituye un capital cultural. Cuestiones como ésta, que atraviesan el lazo social, se transmiten en las instituciones. Cuando este capital cultural se ha erosionado por las condiciones de marginalización y vulnerabilidad, la posibilidad de transmitir algunos dones de la cultura se vuelve un desafío. Con lo cual muchas veces las instituciones parecen reproducir la expulsión de esta población en los diferentes modos de desalojarla.

La tarea de pensar el género como categoría política, de pensarse ellas dentro de un colectivo, pensar las violencias cotidianas como una problemática social que trasciende lo individual-patológico, les permitió correrse de aquella tarea imposible de adecuación a lo que se esperaba de ellas, que bajaba como imperativo, que las aplastaba y frente al cual sólo podían intentar mantenerse invisibles, así como también resistir para mostrar aquello que no se ve, la repetición en la institución del lugar doméstico: mantenerse al margen de la circulación productiva.

Este trabajo que se dieron, el de tomar la 